\title{
Quantitative bone marrow lesion size in osteoarthritic knees correlates with cartilage damage and predicts longitudinal cartilage loss
}

\author{
Jeffrey B Driban ${ }^{1 *}$, Grace H Lo², Ji Yeon Lee ${ }^{1}$, Robert J Ward ${ }^{3}$, Eric Miller ${ }^{4}$, Jincheng Pang ${ }^{4}$, Lori Lyn Price 5 and \\ Timothy E McAlindon ${ }^{1}$
}

\begin{abstract}
Background: Bone marrow lesions (BMLs), common osteoarthritis-related magnetic resonance imaging findings, are associated with osteoarthritis progression and pain. However, there are no articles describing the use of 3dimensional quantitative assessments to explore the longitudinal relationship between BMLs and hyaline cartilage loss. The purpose of this study was to assess the cross-sectional and longitudinal descriptive characteristics of BMLs with a simple measurement of approximate BML volume, and describe the cross-sectional and longitudinal relationships between BML size and the extent of hyaline cartilage damage.

Methods: 107 participants with baseline and 24-month follow-up magnetic resonance images from a clinical trial were included with symptomatic knee osteoarthritis. An 'index' compartment was identified for each knee defined as the tibiofemoral compartment with greater disease severity. Subsequently, each knee was evaluated in four regions: index femur, index tibia, non-index femur, and non-index tibia. Approximate BML volume, the product of three linear measurements, was calculated for each BML within a region. Cartilage parameters in the index tibia and femur were measured based on manual segmentation.
\end{abstract}

Results: BML volume changes by region were: index femur (median [95\% confidence interval of the median]) 0.1 $\mathrm{cm}^{3}\left(-0.5\right.$ to $\left.0.9 \mathrm{~cm}^{3}\right)$, index tibia $0.5 \mathrm{~cm}^{3}\left(-0.3\right.$ to $\left.1.7 \mathrm{~cm}^{3}\right)$, non-index femur $0.4 \mathrm{~cm}^{3}\left(-0.2\right.$ to $\left.1.6 \mathrm{~cm}^{3}\right)$, and nonindex tibia $0.2 \mathrm{~cm}^{3}\left(-0.1\right.$ to $\left.1.2 \mathrm{~cm}^{3}\right)$. Among 44 knees with full thickness cartilage loss, baseline tibia BML volume correlated with baseline tibia full thickness cartilage lesion area $(r=0.63, p<0.002)$ and baseline femur BML volume with longitudinal change in femoral full thickness cartilage lesion area $(r=0.48 p<0.002)$.

Conclusions: Many regions had no or small longitudinal changes in approximate BML volume but some knees experienced large changes. Baseline BML size was associated to longitudinal changes in area of full thickness cartilage loss.

\section{Background}

Knee osteoarthritis (OA) is the most common form of arthritis, accounting for substantial disability in the general population [1]. However, there are many gaps in our knowledge regarding the pathophysiology of this disease. Recent evidence suggests that peri-articular bone changes are integral to knee OA pathophysiology

\footnotetext{
* Correspondence: jdriban@tuftsmedicalcenter.org

'Division of Rheumatology, Tufts Medical Center, 800 Washington Street, Box \#406, Boston, MA 02111, USA

Full list of author information is available at the end of the article
}

and may be important for identifying new OA treatments [2-13].

Bone marrow lesions (BMLs), common OA-related magnetic resonance (MR) imaging findings (Figure 1), are associated with OA progression and pain [2-13]. BMLs have been implicated with increased odds of hyaline cartilage damage and subchondral bone attrition [2-5,7-9,12]. To date, no one has published articles describing the use of 3-dimensional quantitative assessments to explore the longitudinal relationship between BMLs and hyaline cartilage.

\section{Biomed Central}

(c) 2011 Driban et al; licensee BioMed Central Ltd. This is an Open Access article distributed under the terms of the Creative Commons Attribution License (http://creativecommons.org/licenses/by/2.0), which permits unrestricted use, distribution, and reproduction in any medium, provided the original work is properly cited. 


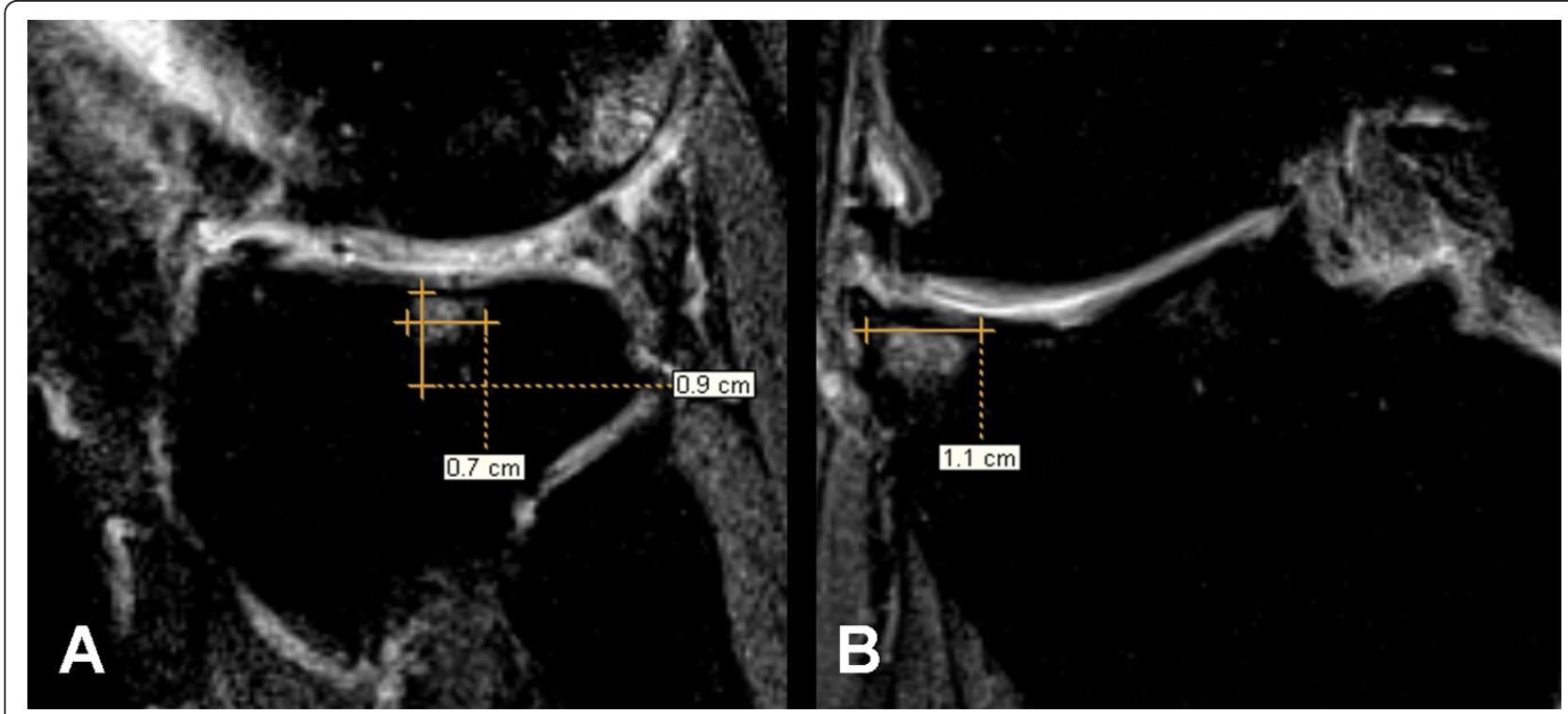

Figure 1 Bone marrow lesion measurements within the lateral tibia. a. In the sagittal image the superior-inferior dimension (0.9 mm) and anterior-posterior dimension $(0.7 \mathrm{~mm})$ are measured. b. In the coronal image the medial-lateral dimension is measured $(1.1 \mathrm{~mm})$. The image contrast is optimized for measuring bone marrow lesions based on the measurement protocol (not for viewing other anatomical structures).

The purpose of this study was to use a simple method of measuring approximate BML volume that uses the product of three linear measurements across images. This method was selected because it does not require proprietary software, requires less time to measure, and provides a continuous measure of approximate BML size. In this manuscript we 1) report cross-sectional and longitudinal descriptive BML characteristics with this new method, and 2) describe the cross-sectional and longitudinal relationships between approximate BML volume and the extent of hyaline cartilage damage using quantitative 3-dimensional measurements.

\section{Methods}

\section{Study sample}

This study was a secondary analysis of a recently completed randomized, placebo-controlled, clinical trial of vitamin D among participants with knee OA $(n=146)$. All participants with both baseline and 24-month follow-up images were included in this study $(n=107$ participants). All participants were required to have knee $\mathrm{OA}$ as defined by the American College of Rheumatology [14] and be over 45 years of age. Additional inclusion criteria were that participants had to have chronic knee discomfort (knee discomfort on most days for at least one month in past 12 months), Western Ontario and McMaster Universities Osteoarthritis Index (WOMAC) pain subscale $\geq 1$, tibiofemoral OA on posterior-anterior weight-bearing semi-flexed knee radiographs (equivalent to Kellgren and Lawrence [KL] grade $\geq 2$ ), and clinical examination confirming knee pain or discomfort referable to the knee joint. To compare the cross-sectional and longitudinal relationships between regional approximate BML volume and the extent of hyaline cartilage damage a sample consisting of 44 knees was selected that only included knees with full thickness cartilage lesions at baseline. This sample is smaller than the full sample because these analyses were restricted to knees with full thickness cartilage lesions to avoid statistical issues associated with more than half of the knees having no areas of full thickness cartilage loss. The Institutional Review Board of Tufts Medical Center approved the study and informed consent was obtained from all participants prior to inclusion.

\section{Knee selection}

One knee was identified as the study knee for each participant. If both knees were eligible, then the knee with a greater WOMAC pain subscale score was selected. If both knees had equivalent WOMAC pain subscale scores, the knee with a greater KL grade was chosen. Finally, if WOMAC pain subscale scores and KL grades were equivalent for both knees, the study knee was randomly selected.

\section{Index compartment selection}

Within each knee, a rheumatologist (TEM) defined an index compartment (medial or lateral tibiofemoral) as the compartment with greater pathology based on radiographs. If the medial and lateral tibiofemoral compartments were comparable then MR images were assessed for cartilage damage, bone marrow lesions, and meniscal 
damage. A second rheumatologist (JYL) independently verified the index compartment by evaluating MR images. If disagreement arose a consensus decision was achieved.

\section{MR image acquisition}

MR images of the study knees were obtained at baseline and at 2 years follow-up on a Siemens Magnetom Avanto 1.5T (Malvern, PA). The sequences of relevance for BML assessment were sagittal and coronal intermediate-weighted (IW) fat-suppressed (FS) images with time to recovery (TR) of $2950 \mathrm{~ms}$, time to echo (TE) of $31 \mathrm{~ms}$, slice thickness of $3 \mathrm{~mm}$, space thickness of 0.5 $\mathrm{mm}$, and field of view (FOV) of $140 \mathrm{~mm}$. The sequences of relevance for cartilage volume assessment were 3dimensional sagittal water excitation dual echo steady state (DESS WE) images with time to recovery (TR) of $18.2 \mathrm{~ms}$, time to echo (TE) of $5.28 \mathrm{~ms}$, slice thickness of $1.3 \mathrm{~mm}$, and field of view (FOV) of $140 \mathrm{~mm}$.

\section{BML assessment}

BMLs were evaluated on IW FS MR images at baseline and 2-years. We defined BMLs as ill-defined areas of high-signal intensity located within $1.0 \mathrm{~cm}$ of articular cartilage and present on either $\geq 2$ sagittal images and/ or $\geq 2$ coronal images and classified them within 4 regions: index femoral region (representing the femoral region of the index compartment), non-index femoral region (representing the femoral region of the opposite compartment), index tibial region, and non-index tibial region. Prior to measuring BML size, image brightness and contrast were adjusted until normal bone signal appeared black and homogenous. Adjustments of image contrast and brightness, as well as BML identification, were conducted on paired images (baseline and 24 months). One reader (JD) measured the maximal anterior-posterior (AP), medial-lateral (ML), and superiorinferior dimension of each BML using the linear measurement tool in eFilm Workstation 3.01 (Merge Healthcare, Milwaukee, WI; Figure 1). AP and superiorinferior dimensions were measured on sagittal images and the ML dimension was determined on coronal images. For each lesion, the linear measurement tool was used to mark the BML edges that defined the greatest diameter for each dimension (e.g. AP, ML) on the sagittal and coronal images with the largest BML crosssection. The line measurements were then copied to all images within the image set and the reader reviewed the adjacent images to determine if the BML extended beyond the measurement lines in any of the other slices. If the BML extended beyond the line then the measurement line was lengthened to represent the maximum width of the BML across images.
We took the product of the three linear measurements to represent the approximate volume of each BML. When multiple BMLs were present in a region (e.g. index femur) their approximate volumes were summed to calculate regional approximate volume. Regional approximate BML volume changes were defined as progression if the volume increased and regression if the volume decreased. We also measured peak signal intensity in each BML on coronal images. Peak signal intensity was normalized to normal bone marrow signal to correct for variations in signal intensity that occurs across MR image acquisitions. Normal bone marrow signal intensity was collected from the posterior non-index femur because this region consistently had no BMLs. When multiple BMLs were present, the BML with greatest signal intensity defined the regional peak signal intensity. Intra-tester reliability (intraclass correlations [ICC 3,1 model])[15] for BML measurements ranged from 0.87 to 0.98 for BML linear measurements; 0.96 and 0.90 for volume and volume change; 0.88 for peak signal intensity. The manually-measured approximate BML volumes were correlated to segmentation BML volumes performed by an independent rater (JP; ICC $[2,1]=0.81, \mathrm{n}=17$ BMLs). Furthermore, a Bland-Altman analysis indicated the $95 \%$ limit of agreement between manual and segmented BML size ranged from -2.8 to $1.3 \mathrm{~cm}^{3}$. Sixteen of the 17 manually-measured approximate BML volumes were in agreement with the segmented BML volume. Manual measurements were always greater than the segmented BML volumes (ranged from -3.8 to $0.0 \mathrm{~cm}^{3}$ ).

\section{Cartilage assessment}

Cartilage parameters were evaluated on sagittal DESS MR images at baseline and 24 months. Using Analyze $8.1^{\odot}$ (Mayo Clinic, Rochester, MN), one investigator (JYL), unblinded to the image acquisition order, registered the baseline and 24 month MR images and then performed paired manual segmentation of the indexcompartment tibia and femur cartilage (Figure 2). Peripheral osteophytes were excluded in image segmentation. After completion of the manual segmentation cartilage volume $\left(\mathrm{mm}^{3}\right)$ was calculated using Analyze (intra-tester ICC > 0.99).

To assess areas of full-thickness cartilage lesions, a second investigator (JBD) used Analyze $8.1^{\odot}$ to place a line in areas of full thickness cartilage lesions (Figure 2). In full thickness cartilage lesions with a central osteophyte the segmentation line was placed through the base of the osteophyte [16]. A MatLab (The MathWork, Natick, MA) custom program (written by EM) then analyzed these images and calculated mean cartilage thickness (with regions of full thickness cartilage lesions 


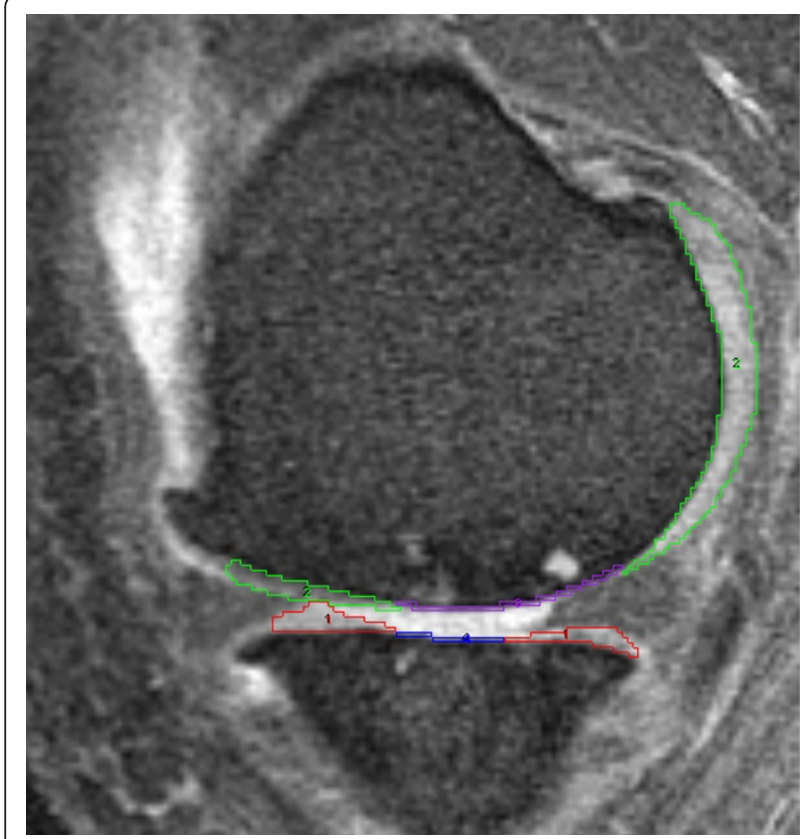

Figure 2 Cartilage segmentation (green and red outlines) with lines (purple and blue) in areas of a full thickness cartilage lesion.

represented as $0 \mathrm{~mm}$ ) as well as areas of full thickness cartilage lesions.

The custom MatLab program used the pixels identified as cartilage in each image. Binary morphological processing methods $[17,18]$ were employed to determine the mean cartilage thickness. For each image slice the thickness of each connected component (CC) of the cartilage was computed as the mean distance from each pixel on the boundary of that $\mathrm{CC}$ from the morphological skeleton (a generalization of the centerline to arbitrary shapes) of that CC. This mean cartilage thickness was computed as twice the total distance to the skeleton of all points along the perimeter divided by the number of points times the linear size of each voxel.

To determine the length of full thickness cartilage lesions in each MR image slice the following process was undertaken. First, the morphological skeletons were computed for each pair of connected cartilage segments that was separated by a full thickness cartilage lesion. A second order polynomial was fit to the points from both morphological skeletons resulting in a curve that "spanned" the full thickness cartilage lesion between the two pieces of cartilage. The portion of this curve not overlapping the two cartilage pieces was taken to be the length of the full thickness cartilage lesion. To determine the mean cartilage thickness including the full thickness cartilage lesion, we estimated the number of pixels that comprise the full thickness cartilage lesion as the product of the full thickness cartilage lesion length and the density of pixels (pixels per unit length) as computed from the two bordering cartilage segments. The mean cartilage thickness including the full thickness cartilage lesion in each slice was then computed as previously described, but the number of points in the denominator was the sum of the number of pixels associated with both the full thickness cartilage lesion and cartilage segments. The mean cartilage thickness (with regions of full thickness cartilage lesions represented as $0 \mathrm{~mm}$ ) was averaged across slices.

Finally, the area of full thickness cartilage lesion was computed as the ratio of the area of the full thickness cartilage lesion to the area of the full thickness cartilage lesion plus the surface area of the cartilage pieces (total area of subchondral bone) where the latter was calculated as the curve length of the second order polynomial that did intersect the two pieces of cartilage $[17,18]$.

\section{Statistical analyses}

Descriptive data were calculated for the study sample. Change in approximate BML volume was classified as regression (getting smaller), progression (getting larger), or no change in size relative to measurement error (test, retest). Longitudinal BML measurement error was calculated with the reliability data set (described above) by calculating the difference between the first approximate BML volume change and the retest approximate BML volume change (Differences in approximate BML volume change $=\left[\right.$ Follow-up $1-$ Baseline $\left._{1}\right]-[$ Follow-up 2 - Baseline ${ }_{2}$ ]). Smallest detectable differences were originally calculated based on two standard deviations of the differences in approximate BML volume change (i.e., $95 \%$ confidence interval [95\% CI] of the mean), however the data were not normally distributed and the sample size was small $\left(n=16\right.$ BMLs, mean $=1.86 \mathrm{~cm}^{3}$, median $=0.4 \mathrm{~cm}^{3}$, standard deviation $=5.99 \mathrm{~cm}^{3}$ ). Therefore, the smallest detectable difference was derived from the $95 \% \mathrm{CI}$ of the median (median $=0.4 \mathrm{~cm}^{3} ; 95 \% \mathrm{CI}=-0.1$ to $3.2 \mathrm{~cm}^{3}$ ). To be conservative, we chose the larger endpoint of the $95 \% \mathrm{CI}$ of the median. BML regression was defined as an approximate BML change less than $-3.2 \mathrm{~cm}^{3}$ over 2 years and BML progression was defined as an approximate BML change greater than $3.2 \mathrm{~cm}^{3}$ over 2 years (changes between $-3.2 \mathrm{~cm}^{3}$ and $3.2 \mathrm{~cm}^{3}$ were classified as no change). To further explore this definition of change we evaluated a boot-strapping resampling method (1000 resamples). This method and the $95 \% \mathrm{CI}$ of the median resulted in a similar distribution for classifying BMLs as regression, no change, and progression.

Regional BML cross-sectional and longitudinal descriptive characteristics were limited to participants with BMLs present in the specific region (e.g. index femur). We used Spearman correlations to evaluate the 
intra-regional associations between regional baseline and longitudinal BML volume as well as regional approximate BML volume and extent of cartilage damage. These correlations were limited to a sample of knees with full thickness cartilage lesions at baseline (see the Study Sample section for justification). Fisher's z transformations were performed to determine $95 \%$ CI for correlation coefficients. To assess the external validity of the correlations between approximate BML volume and extent of cartilage damage, Spearman correlations were analyzed, using the entire cohort $(n=107)$, to evaluate the intra-regional associations between regional approximate BML volume, cartilage volume, and cartilage thickness. Differences in correlation coefficients between the entire cohort and subset with full thickness cartilage lesions were defined by coefficients being within the 95\% confidence intervals of the correlation coefficient that it was being compared. Bonferroni corrections were used to correct for the 24. Spearman correlations that evaluated the hypotheses that approximate BML volumes were associated with cartilage damage (adjusted $\mathrm{p}$ value for significance was $\mathrm{p}<0.002$ ). Significant univariate correlations were explored with follow-up stepwise multiple linear regression models to determine if potential covariates (i.e., age, sex, body mass index, intervention group) influenced the associations.

Within the index compartment structural changes in the femur or tibia may alter the loading throughout the compartment. Therefore, exploratory Spearman correlations evaluated the inter-regional relationships within the index compartment between regional approximate BML volume and extent of cartilage damage. We used SAS 9.2 (SAS Institute, Cary, NC) to calculate regional approximate BML volumes, derive median and 95\% CI for the median as well as perform all statistical analyses.

\section{Results}

Descriptive characteristics of regional approximate BML volume and signal intensity

From the original trial, 107 participants were included in the analyses with a mean age of $63 \pm 9$ years, mean body mass index of $29.8 \pm 5.4 \mathrm{~kg} / \mathrm{m}^{2}, 64 \%$ female; and $52 \%$ KL grade 2 (56 knees),30\% KL grade 3 (32 knees), and $18 \%$ KL 4 grade (19 knees). In this sample 101
(94\%) knees had $\geq 1$ BML (range 1 to 7) for a total of 240 BMLs. Furthermore, 45 (19\%) BMLs regressed (got smaller), 61 (25\%) BMLs progressed (got larger), and 134 (56\%) did not change over two years. Table 1 provides regional baseline approximate BML volumes. While most regions demonstrated no or small regional approximate BML volume changes over 2 years, some underwent large changes (Figure 3, Table 1). Longitudinal changes in regional approximate BML volume were not related to baseline approximate BML volumes (Table 1; see scatter plots in Additional File 1).

\section{Relationships between approximate BML volume and cartilage parameters within index compartments}

To compare the cross-sectional and longitudinal relationships between regional approximate BML volume and the extent of hyaline cartilage damage, a sample consisting of 44 knees was selected ( $65 \pm 9$ years of age (mean \pm standard deviation), $52 \%$ female, and body mass index of $29.8 \pm 5.8 \mathrm{~kg} / \mathrm{m}^{2}$ ). Among index femurs, $91 \%$ had a full thickness cartilage lesion at baseline (baseline cartilage lesion $=12.1 \pm 10.3 \%$ of subchondral bone; 2 knees developed a full thickness cartilage lesion when none was present at baseline; change $=1.5 \pm 1.8 \%$ of subchondral bone) and 91\% had baseline BMLs (baseline BML size = $15.3 \pm 16.0 \mathrm{~cm}^{3}$; 1 knee developed a BML when none was present at baseline; 4 knees had a BML at baseline that was absent at follow-up; change $=1.6 \pm 12.4 \mathrm{~cm}^{3}$ ). Among index tibiae, $66 \%$ had a full thickness cartilage lesion at baseline (baseline cartilage lesion $=24.8 \pm 17.8 \%$ of subchondral bone; 3 knees developed a full thickness cartilage lesion when none was present at baseline; change $=3.0 \pm 4.7 \%$ of subchondral bone) and $84 \%$ had baseline BMLs (baseline BML size $=30.7 \pm 31.5 \mathrm{~cm}^{3} ; 2$ knees developed a BML when none was present at baseline; change $=3.9 \pm 14.8 \mathrm{~cm}^{3}$ ).

Table 2 describes the intra-regional correlations, in the index compartment, between cartilage and regional approximate BML volume (see scatter plots in Additional File 1). After Bonferroni adjustments for multiple testing only two statistically significant correlations were present between regional approximate BML volume and cartilage parameters: baseline tibia BML volume to baseline tibia full thickness cartilage lesion area and baseline

Table 1 Median (95\% Cl) Baseline and Longitudinal Changes in Approximate BML Volume

\begin{tabular}{lccc}
\hline Region & Baseline BML Volume $\left(\mathbf{c m}^{\mathbf{3}}\right)$ & BML Volume Change $\left.\mathbf{( c m}^{\mathbf{3}}\right)$ & $\begin{array}{c}\text { Spearman Correlation }(\mathbf{9 5 \%} \mathbf{C l}): \\
\text { Baseline to Longitudinal Change }\end{array}$ \\
\hline Index Femur $(n=76)$ & $4.2(2.5$ to 11.5$)$ & $0.1(-0.5$ to 0.9$)$ & $-0.20(-0.41$ to 0.02$)$ \\
Index Tibia $(n=76)$ & $8.8(3.9$ to 14.3$)$ & $0.5(-0.3$ to 1.7$)$ & $-0.14(-0.35$ to 0.09$)$ \\
Non-index Femur $(n=33)$ & $2.6(1.6$ to 8.2$)$ & $0.4(-0.2$ to 1.6$)$ & $-0.06(-0.40$ to 0.29$)$ \\
Non-index Tibia $(n=30)$ & $1.1(0.6$ to 3.9$)$ & $0.2(-0.1$ to 1.2$)$ & $-0.27(-0.57$ to 0.10$)$ \\
\hline
\end{tabular}

No Spearman correlation coefficients were statistically significant. 95\% Cl =95\% confidence interval of the median or 95\% confidence interval of the Spearman Correlation. See Additional File 1 for scatter plots of the analyzed correlations. 

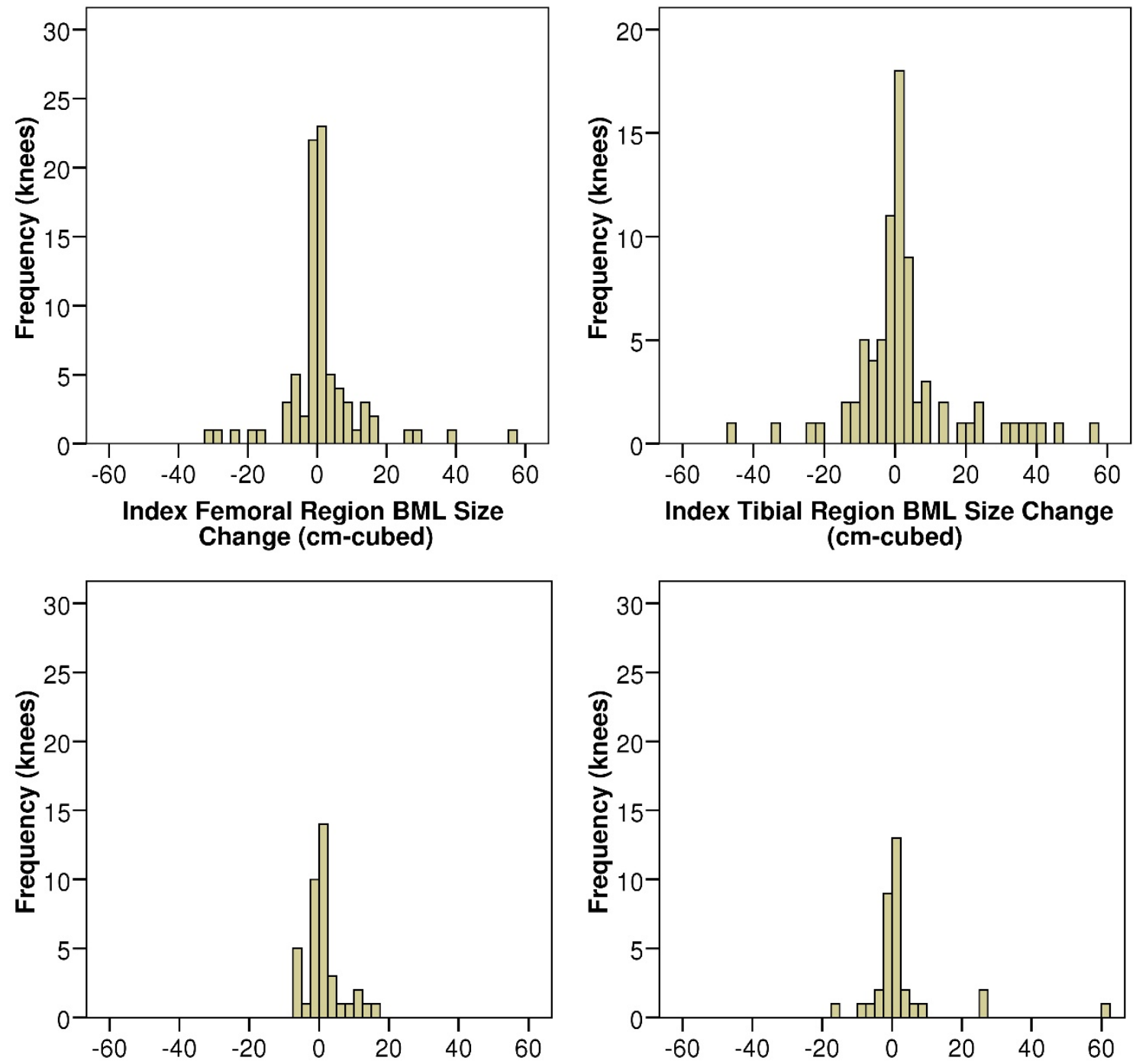

Non-Index Femoral Region BML Size Change (cm-cubed)

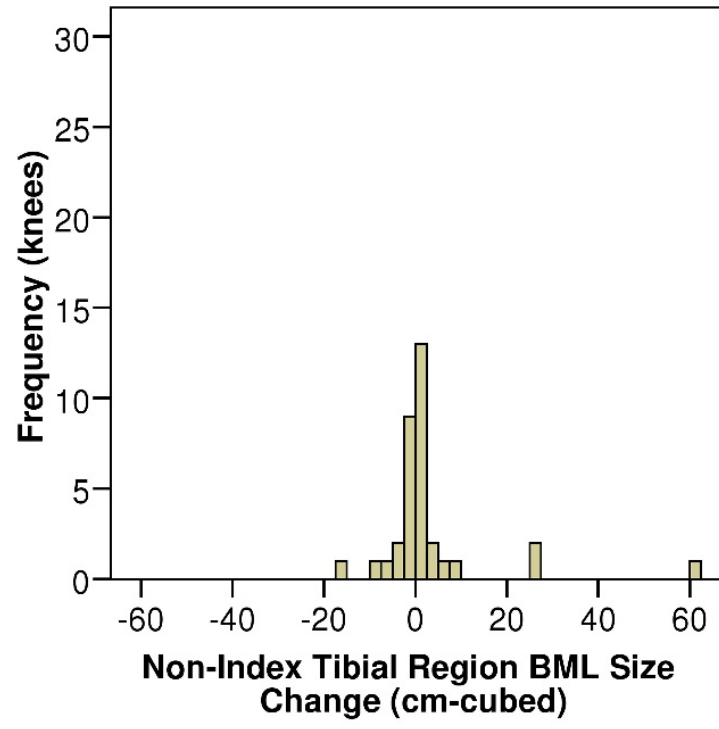

Figure 3 Histograms of bone marrow lesion (BML) size change among regions with BMLs. Each bar represents a range of $2.5 \mathrm{~cm}^{3}$.

femur BML volume to change in femoral full thickness cartilage lesion area. There were no other statistically significant correlations between cartilage parameters (baseline or change) and BML volume change. To assess the external validity of these findings, approximate BML volume and extent of cartilage damage correlations between approximate BML volume, cartilage volume, and cartilage thickness were evaluated and determined to be similar between the subset $(n=44)$ and entire cohort ( $n=107$; see table in Additional File 2). Based on stepwise multiple linear regressions age, sex, body mass index, or intervention group did not influence significant univariate associations.
Exploratory correlations between index-compartment regions were examined: index-femoral baseline BML with index-tibial baseline full thickness cartilage lesion area $(r$ $=0.40, p=0.01 ; n=40)$, index-femoral baseline BML with index-tibial full thickness cartilage lesion area change $(r=0.39, p=0.01 ; n=40)$, index-tibial baseline BML with index-femoral baseline full thickness cartilage lesion area $(r=0.55, p<0.001, n=37)$, index-tibial BML change with index-femoral baseline cartilage volume $(r=$ -0.33, $p=0.04, n=39$ ), and index-tibial BML change with index-femoral baseline cartilage thickness $(r=-0.38$, $p=0.02, n=39$ ). No other statistically significant correlations were detected across regions of the index. 
Table 2 Within-Region Spearman Coefficients (95\% Confidence Intervals) Among Baseline BML and Cartilage Parameters in the Index Compartment

\begin{tabular}{|c|c|c|c|c|}
\hline & $\begin{array}{l}\text { Femur BML Volume: } \\
\text { Baseline } \\
(n=40)\end{array}$ & $\begin{array}{c}\text { Tibia BML Volume: } \\
\text { Baseline } \\
(n=37)\end{array}$ & $\begin{array}{c}\text { Femur BML Volume: } \\
\text { Change } \\
(n=41)\end{array}$ & $\begin{array}{c}\text { Tibia BML Volume: } \\
\text { Change } \\
(n=39)\end{array}$ \\
\hline \multicolumn{5}{|l|}{ Baseline } \\
\hline Cartilage Volume & $-0.06(-0.37,0.25)$ & $-0.31(-0.57,0.02)$ & $-0.06(-0.37,0.25)$ & $-0.08(-0.39,0.24)$ \\
\hline Cartilage Thickness & $0.02(-0.30,0.33)$ & $-0.37(-0.62,-0.05)$ & $-0.12(-0.42,0.19)$ & $-0.02(-0.33,0.30)$ \\
\hline $\begin{array}{l}\text { Full Thickness Cartilage Lesion } \\
\text { Area }\end{array}$ & $0.33(0.02,0.58)$ & $0.63(0.38,0.79)^{*}$ & $-0.06(-0.36,0.26)$ & $-0.03(-0.34,0.29)$ \\
\hline \multicolumn{5}{|l|}{ 2-year Longitudinal Change } \\
\hline Cartilage Volume & $0.03(-0.29,0.34)$ & $0.16(-0.18,0.46)$ & $-0.10(-0.39,0.22)$ & $0.32(0.00,0.58)$ \\
\hline Cartilage Thickness & $-0.11(-0.41,0.21)$ & $-0.13(-0.43,0.21)$ & $-0.15(-0.43,0.17)$ & $0.04(-0.28,0.35)$ \\
\hline $\begin{array}{l}\text { Full Thickness Cartilage Lesion } \\
\text { Area }\end{array}$ & $0.48(0.20,0.69)^{*}$ & $0.43(0.12,0.66)$ & $0.08(-0.23,0.38)$ & $-0.18(-0.47,0.14)$ \\
\hline
\end{tabular}

* statistically significant finding after Bonferroni corrections (24 multiple comparisons; $\mathrm{p}<.002$; see Additional File 1 for scatter plots).

\section{Discussion}

This study used a simple and novel method of measuring approximate BML volume that can be easily employed by researchers and clinicians. The technique demonstrated that many knee regions experienced small longitudinal changes in regional approximate $\mathrm{BML}$ volume over 2 years $\left( \pm 2.5 \mathrm{~cm}^{3}\right)$ but some knees experienced large longitudinal changes. In agreement with previous findings, baseline regional approximate BML volume and hyaline cartilage full-thickness lesion area (baseline and longitudinal change) were associated $[2,4,5,7,8,12,19-28]$. The new BML measurement technique is sensitive to small changes in BML size over 2 years and, in agreement with previous literature, positively associated with increased cartilage damage.

To our knowledge, this is the first full-length publication reporting the longitudinal relationship between 3dimensional quantitative measurements of BML size and hyaline cartilage among knees with OA. Several methods have been deployed to quantify BML size but they have various limitations. One method uses manual segmentation of all MR image slices that display a BML. This method is time consuming and usually requires proprietary software [29-36]. Therefore, a segmentation technique is difficult to deploy researchers working with large cohorts. A less labor-intensive method that has been used is to take the measurement of the greatest diameter of a lesion. One potential downside to this approach is that it does not account for the threedimensional nature of these lesions [7]. An alternative method that is not very labor intensive approximates BML volume with three linear measurements; specifically, the coronal and sagittal images with the largest area of signal abnormality are identified and the largest three dimensions from these two images $[37,38]$. While this appears to be a good compromise between function and practicality, this method does not take into account for the possibility that the greatest width of a BML may change as you view adjacent coronal or sagittal images (Figure 4). By measuring the greatest diameter across images the current technique attempts to account for the potential limitations that a single image may not adequately describe the 3-dimensional size of an irregularly shaped BML.

Baseline regional approximate BML volume is positively associated with baseline and longitudinal changes in full thickness cartilage lesion area. A positive correlation between semiquantitative assessments of BML size and cartilage damage have been demonstrated in crosssectional [4,20-24] and longitudinal studies $[2,4,5,8,19,24,25]$. Previous reports regarding the withinregion association between $B M L$ size and cartilage volume, the most common quantitative measure of hyaline cartilage, are conflicting with some showing statistically significant associations [7,12,21,24,26-28] and others showing non-significant associations $[2,7,20,21,25,27]$. The current correlations concur with previously reported associations between longitudinal change of BML size (greatest diameter) and cartilage volume ( $r=-0.03$ to -0.40$)$ [7]. Furthermore, one article, which distinguished between full thickness cartilage lesions and normal or partial thickness lesions, noted that only $7 \%$ of knees (11/149 knees) with normal cartilage or partial thickness defect had a BML greater than $1 \mathrm{~cm}$ in size while $33 \%$ of knees (16/48 knees) with a full thickness defect had a BML greater than $1 \mathrm{~cm}$ [10]. These data support the current findings that BML size may have a strong association with full thickness cartilage lesion area.

Baseline regional approximate BML volume was statistically associated with baseline and longitudinal changes in full thickness cartilage lesion area but not cartilage thickness or volume. The similar findings between cartilage thickness and volume are not unexpected since the 


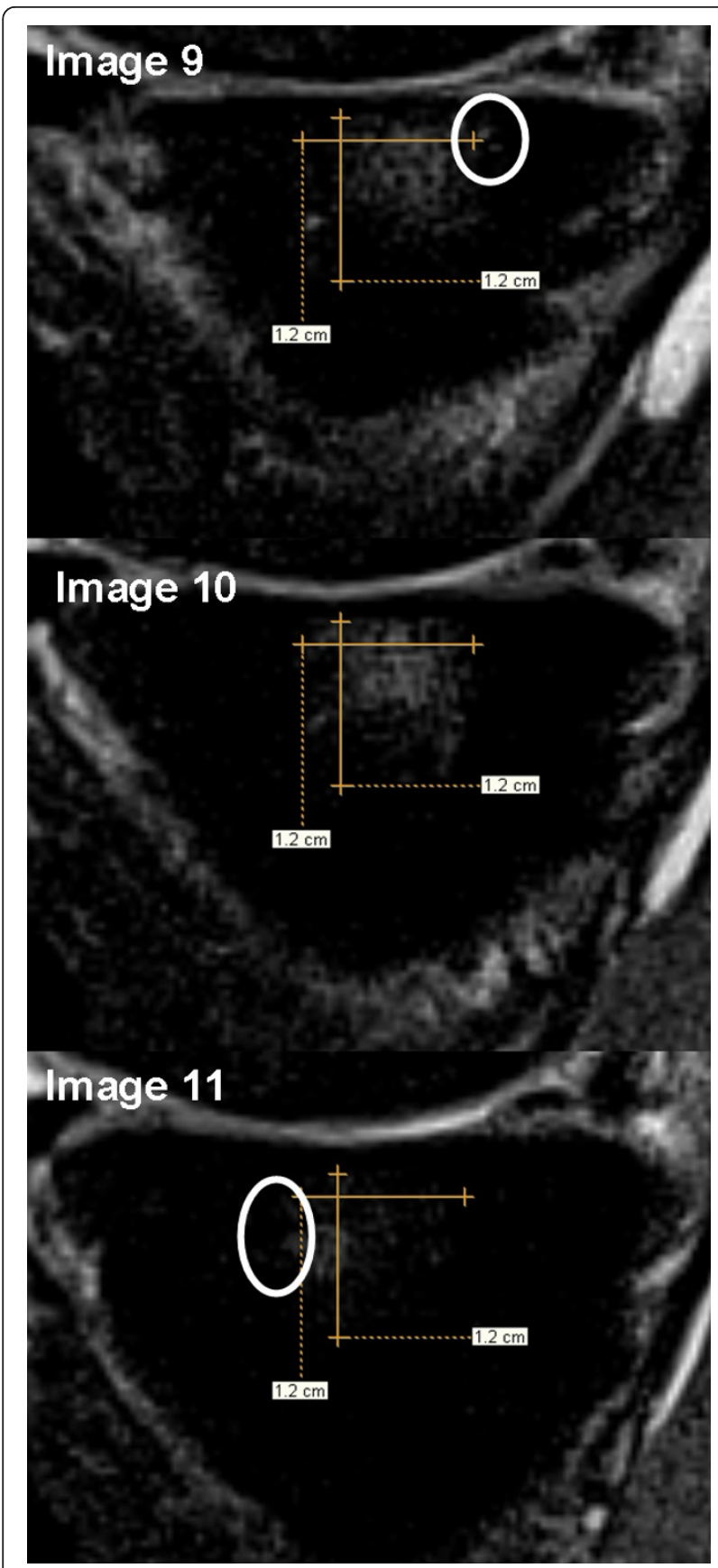

Figure 4 Measurements of approximate BML volume based on only the coronal and sagittal images with the largest area of signal abnormality may misrepresent the greatest diameter of a BML. Image 10, image with the largest BML area, would traditionally be measured to approximate BML volume and result in an anterior-posterior diameter of $1.2 \mathrm{~cm}$ but with the newer technique, measuring across images (including images 9 and 11), the anterior-posterior diameter is $1.5 \mathrm{~cm}$. The white circles indicate areas of increased signal intensity beyond the dimensions indicated on image 10. The image contrast is optimized for measuring bone marrow lesions based on the measurement protocol (not for viewing other anatomical structures) parameters are based on similar cartilage data (e.g. cartilage thickness determines cartilage volume). Although the design of this study did not allow us to determine why regional BML volumes had stronger correlations with full thickness cartilage lesion area compared to cartilage volume or thickness, we speculate that BMLs are an early and sensitive biomarker of localized degeneration in response to altered loading. Furthermore, BMLs may also contribute to localized cartilage loss leading to full thickness cartilage lesions. A second hypothesis is that BMLs have a localized influence on cartilage loss and full thickness cartilage lesion area is a more localized measure of hyaline cartilage loss than the other two measures. These hypotheses are supported by the associations between baseline BML size and longitudinal changes in full thickness cartilage lesion area. A third hypothesis is that the association between BML size and cartilage volume and thickness is impeded because OA progression defined by cartilage thickness and volume is not always linear. Some patients experience increased cartilage thickness with KL grade 2 (23\% of knees) and KL grade $3(18 \%)$ while the majority lose cartilage thickness and volume [39]. In contrast, full thickness cartilage lesion area progresses in a linear pattern, which supports its higher correlations with BML size.

Exploratory analyses across regions demonstrated statistically significant associations including relations between baseline cartilage and regional approximate BML volume change. When assessing predictors of longitudinal cartilage loss or BML volume change it is important to consider other regions. Altered structure in one region of the index compartment is likely to alter the loading in both index regions. Inter-regional associations between BML and cartilage parameters warrant its own research. Few studies have evaluated inter-region relationships between BMLs and cartilage parameters but there is previous research to support these relationships. Statistically significant inter-regional correlations between baseline BML size (semi-quantitative) and cartilage volume change have been reported [28]. When accounting for variables that predict disease progression (e.g. cartilage loss, BML progression) it may be important to consider the influence of other regions throughout the joint.

Our study showed that small changes in BML size were common but that there is a large variation among knees with OA. This corroborates findings from other investigators who also found that among OA knees with BMLs, there was a large variability of longitudinal BML size change $[7,36]$. In a small sample $(n=14)$, investigators using semi-automated BML segmentation showed that $43 \%$ increased BML volume (change greater than $5 \%), 36 \%$ decreased BML volume, and $21 \%$ did not change ( $\pm 5 \%$ change)[36]. Small longitudinal changes 
with large variability has also been reported with assessments of maximum BML diameter (i.e. coefficient of variation $[\mathrm{CV}]=719 \%, 4,360 \%)[7]$.

While the findings of the current study are interesting, there are several limitations. Approximate BML volume over-estimates the true BML volume but may be strongly correlated to the true BML volume. Furthermore, the method may not detect subtle changes within the baseline approximate volume.

Our data support the construct validity of the new methodology. This technique is intended to be a simple and efficient method of measuring 3-dimensional BML size that can be done with standard MR imaging viewing software. Future research should determine the relationship between true BML volume determined through BML segmentation and approximate BML volume using this technique.

\section{Conclusions}

In summary, this study demonstrates a method of approximating BML volume that can be readily adopted by clinicians and researchers. The measurements detected longitudinal changes during a two-year clinical trial. Furthermore, baseline BML size was associated to the longitudinal changes in full thickness cartilage lesion area. More research is warranted to investigate the longitudinal relationship between quantitative measures of tissue degeneration associated with OA.

\section{Additional material}

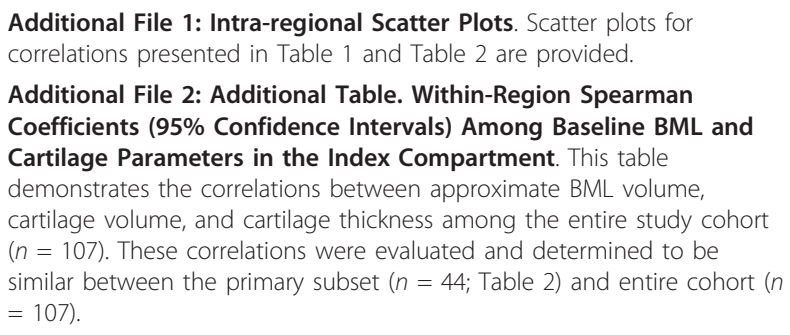

\section{Acknowledgements}

Timothy E. McAlindon received support from National Institute of Arthritis and Musculoskeletal and Skin Diseases (R01AR051361-04).

\footnotetext{
Author details

'Division of Rheumatology, Tufts Medical Center, 800 Washington Street, Box \#406, Boston, MA 02111, USA. ${ }^{2}$ Section of Immunology, Allergy, and Rheumatology, Michael E. DeBakey VAMC, and Baylor College of Medicine, 1 Baylor Plaza, BCM-285, Houston, TX 77030, USA. ${ }^{3}$ Department of Radiology, Tufts Medical Center, 800 Washington Street, Box \#299, Boston, MA 02111, USA. ${ }^{4}$ Department of Electrical and Computer Engineering, Tufts University, 216 Halligan Hall, Medford, MA 02155, USA. ${ }^{5}$ Biostatistics Research Center, Institute for Clinical Research and Health Policy Studies, Tufts Medical Center, 800 Washington Street, Box \#63, Boston, MA 02111, USA.
}

\section{Authors' contributions}

JBD contributed to the conception and design, collection and assembly of data, analysis and interpretation of data, drafting/revisions of article, as well as final approval of the article.

$\mathrm{GL}$ contributed to the analysis and interpretation of data, drafting/revisions of article, as well as final approval of the article. JYL contributed to the collection and assembly of data, revisions of article, as well as final approval of the article. RW contributed to the conception and design, revisions of article, as well as final approval of the article. EM contributed to the collection and assembly of data, analysis and interpretation of data, revisions of article, as well as final approval of the article. JP contributed to the collection and assembly of data, revisions of article, as well as final approva of the article. LLP contributed to the conception and design, assembly of data, analysis and interpretation of data, drafting/revisions of article, as well as final approval of the article. TEM contributed to the conception and design, analysis and interpretation of data, obtaining funding, drafting/ revisions of article, as well as final approval of the article.

\section{Competing interests}

The authors declare that they have no competing interests.

Received: 30 June 2011 Accepted: 30 September 2011

Published: 30 September 2011

\section{References}

1. Lawrence RC, Felson DT, Helmick CG, Arnold LM, Choi H, Deyo RA, Gabriel S, Hirsch R, Hochberg MC, Hunder GG, et al: Estimates of the prevalence of arthritis and other rheumatic conditions in the United States: Part II. Arthritis Rheum 2008, 58:26-35.

2. Davies-Tuck M, Wluka AE, Forbes A, Wang Y, English DR, Giles GG, O'Sullivan R, Cicuttini FM: Development of bone marrow lesions is associated with adverse effects on knee cartilage while resolution is associated with improvement - a potential target for prevention of knee osteoarthritis: a longitudinal study. Arthritis Res Ther 2010, 19(12):R10.

3. Hernandez-Molina G, Guermazi A, Niu J, Gale D, Goggins J, Amin S, Felson DT: Central bone marrow lesions in symptomatic knee osteoarthritis and their relationship to anterior cruciate ligament tears and cartilage loss. Arthritis Rheum 2008, 58:130-136.

4. Hunter DJ, Lo GH, Gale D, Grainger AJ, Guermazi A, Conaghan PG: The reliability of a new scoring system for knee osteoarthritis MRI and the validity of bone marrow lesion assessment: BLOKS (Boston Leeds Osteoarthritis Knee Score). AnnRheum Dis 2008, 67:206-211.

5. Kothari A, Guermazi A, Chmiel JS, Dunlop D, Song J, Almagor O, Marshall M, Cahue S, Prasad P, Sharma L: Within-subregion relationship between bone marrow lesions and subsequent cartilage loss in knee osteoarthritis. Arthritis Care Res 2010, 62:198-203.

6. Lo GH, McAlindon TE, Niu J, Zhang Y, Beals C, Dabrowski C, Le Graverand MP, Hunter DJ: Bone marrow lesions and joint effusion are strongly and independently associated with weight-bearing pain in knee osteoarthritis: data from the osteoarthritis initiative. Osteoarthritis Cartilage 2009, 17:1562-1569.

7. Raynauld JP, Martel-Pelletier J, Berthiaume MJ, Abram F, Choquette D, Haraoui B, Beary JF, Cline GA, Meyer JM, Pelletier JP: Correlation between bone lesion changes and cartilage volume loss in patients with osteoarthritis of the knee as assessed by quantitative magnetic resonance imaging over a 24-month period. AnnRheum Dis 2008, 67:683-688.

8. Roemer FW, Guermazi A, Javaid MK, Lynch JA, Niu J, Zhang Y, Felson DT, Lewis CE, Torner J, Nevitt MC: Change in MRI-detected subchondral bone marrow lesions is associated with cartilage loss: the MOST Study. A longitudinal multicentre study of knee osteoarthritis. AnnRheum Dis 2009, 68:1461-1465.

9. Roemer FW, Neogi T, Nevitt MC, Felson DT, Zhu Y, Zhang Y, Lynch JA, Javaid MK, Crema MD, Torner J, et al: Subchondral bone marrow lesions are highly associated with, and predict subchondral bone attrition longitudinally: the MOST study. Osteoarthritis Cartilage 2010, 18:47-53.

10. Sowers MF, Hayes C, Jamadar D, Capul D, Lachance L, Jannausch M, Welch G: Magnetic resonance-detected subchondral bone marrow and cartilage defect characteristics associated with pain and X-ray-defined knee osteoarthritis. Osteoarthritis Cartilage 2003, 11:387-393. 
11. Torres L, Dunlop DD, Peterfy C, Guermazi A, Prasad P, Hayes KW, Song J, Cahue S, Chang A, Marshall M, Sharma L: The relationship between specific tissue lesions and pain severity in persons with knee osteoarthritis. Osteoarthritis Cartilage 2006, 14:1033-1040.

12. Tanamas SK, Wluka AE, Pelletier JP, Pelletier JM, Abram F, Berry PA, Wang Y, Jones G, Cicuttini FM: Bone marrow lesions in people with knee osteoarthritis predict progression of disease and joint replacement: a longitudinal study. Rheumatology (Oxford) 2010, 49:2413-2419.

13. Yusuf E, Kortekaas MC, Watt I, Huizinga TW, Kloppenburg M: Do knee abnormalities visualised on MRI explain knee pain in knee osteoarthritis? A systematic review. Ann Rheum Dis 2011, 70:60-67.

14. Altman R, Asch E, Bloch D, Bole G, Borenstein D, Brandt K, Christy W, Cooke TD, Greenwald R, Hochberg M: Development of criteria for the classification and reporting of osteoarthritis. Classification of osteoarthritis of the knee. Diagnostic and Therapeutic Criteria Committee of the American Rheumatism Association. Arthritis Rheum 1986, 29:1039-1049.

15. Shrout PE, Fleiss JL: Intraclass correlations: uses in assessing rater reliability. Psychol Bull 1979, 86:420-428.

16. Eckstein F, Ateshian G, Burgkart R, Burstein D, Cicuttini F, Dardzinski B, Gray M, Link TM, Majumdar S, Mosher T, et al: Proposal for a nomenclature for magnetic resonance imaging based measures of articular cartilage in osteoarthritis. Osteoarthritis Cartilage 2006, 14:974-983.

17. Gonzalez RC, Woods RE: Representation and Description. In Digital Image Processing. Volume 3. Upper Saddle River, NJ: Prentice Hall; 2008:795-860.

18. Gonzalez RC, Woods RE: Morphological Image Processing. In Digital Image Processing. Volume 3. Upper Saddle River, NJ: Prentice Hall; 2008:627-688.

19. Hunter DJ, Zhang Y, Niu J, Goggins J, Amin S, LaValley MP, Guermazi A, Genant H, Gale D, Felson DT: Increase in bone marrow lesions associated with cartilage loss: a longitudinal magnetic resonance imaging study of knee osteoarthritis. Arthritis Rheum 2006, 54:1529-1535.

20. Baranyay FJ, Wang Y, Wluka AE, English DR, Giles GG, Sullivan RO, Cicuttini FM: Association of bone marrow lesions with knee structures and risk factors for bone marrow lesions in the knees of clinically healthy, community-based adults. Semin Arthritis Rheum 2007, 37:112-118.

21. Guymer E, Baranyay F, Wluka AE, Hanna F, Bell RJ, Davis SR, Wang Y, Cicuttini FM: A study of the prevalence and associations of subchondral bone marrow lesions in the knees of healthy, middle-aged women. Osteoarthritis Cartilage 2007, 15:1437-1442.

22. Peterfy CG, Guermazi A, Zaim S, Tirman PF, Miaux Y, White D, Kothari M, Lu Y, Fye K, Zhao S, Genant HK: Whole-Organ Magnetic Resonance Imaging Score (WORMS) of the knee in osteoarthritis. Osteoarthritis Cartilage 2004, 12:177-190.

23. Scher C, Craig J, Nelson F: Bone marrow edema in the knee in osteoarthrosis and association with total knee arthroplasty within a three-year follow-up. Skeletal Radiol 2008, 37:609-617.

24. Wluka AE, Hanna F, vies-Tuck M, Wang Y, Bell RJ, Davis SR, Adams J, Cicuttini FM: Bone marrow lesions predict increase in knee cartilage defects and loss of cartilage volume in middle-aged women without knee pain over 2 years. AnnRheum Dis 2009, 68:850-855.

25. Wluka AE, Wang Y, vies-Tuck M, English DR, Giles GG, Cicuttini FM: Bone marrow lesions predict progression of cartilage defects and loss of cartilage volume in healthy middle-aged adults without knee pain over 2 yrs. Rheumatology (Oxford) 2008, 47:1392-1396.

26. Davies-Tuck ML, Wluka AE, Forbes A, Wang Y, English DR, Giles GG, Cicuttini F: Smoking is associated with increased cartilage loss and persistence of bone marrow lesions over 2 years in community-based individuals. Rheumatology (Oxford) 2009, 48:1227-1231.

27. Wildi LM, Raynauld JP, Martel-Pelletier J, Abram F, Dorais M, Pelletier JP: Relationship between bone marrow lesions, cartilage loss and pain in knee osteoarthritis: results from a randomised controlled clinical trial using MRI. AnnRheum Dis 2010, 69:2118-2124.

28. Pelletier JP, Raynauld JP, Berthiaume MJ, Abram F, Choquette D, Haraoui B, Beary JF, Cline GA, Meyer JM, Martel-Pelletier J: Risk factors associated with the loss of cartilage volume on weight-bearing areas in knee osteoarthritis patients assessed by quantitative magnetic resonance imaging: a longitudinal study. Arthritis Res Ther 2007, 9:R74.

29. Anandarajah AP, Schwarz EM, Totterman S, Monu J, Feng CY, Shao T, HaasSmith SA, Ritchlin CT: The effect of etanercept on osteoclast precursor frequency and enhancing bone marrow oedema in patients with psoriatic arthritis. AnnRheum Dis 2008, 67:296-301.
30. Frobell RB, Le Graverand MP, Buck R, Roos EM, Roos HP, Tamez-Pena J, Totterman S, Lohmander LS: The acutely ACL injured knee assessed by MRI: changes in joint fluid, bone marrow lesions, and cartilage during the first year. Osteoarthritis Cartilage 2009, 17:161-167.

31. Li X, Ma BC, Bolbos RI, Stahl R, Lozano J, Zuo J, Lin K, Link TM, Safran M, Majumdar S: Quantitative assessment of bone marrow edema-like lesion and overlying cartilage in knees with osteoarthritis and anterior cruciate ligament tear using MR imaging and spectroscopic imaging at 3 Tesla. JMagn Reson/maging 2008, 28:453-461.

32. Mayerhoefer ME, Breitenseher M, Hofmann S, Aigner N, Meizer R, Siedentop H, Kramer J: Computer-assisted quantitative analysis of bone marrow edema of the knee: initial experience with a new method. AJR AmJRoentgenol 2004, 182:1399-1403.

33. Mayerhoefer ME, Breitenseher MJ, Kramer J, Aigner N, Norden C, Hofmann S: STIR vs. T1-weighted fat-suppressed gadolinium-enhanced $\mathrm{MRI}$ of bone marrow edema of the knee: computer-assisted quantitative comparison and influence of injected contrast media volume and acquisition parameters. JMagn Reson/maging 2005, 22:788-793.

34. Mayerhoefer ME, Kramer J, Breitenseher MJ, Norden C, Vakil-Adli A, Hofmann S, Meizer R, Siedentop H, Landsiedl F, Aigner N: Short-term outcome of painful bone marrow oedema of the knee following oral treatment with iloprost or tramadol: results of an exploratory phase II study of 41 patients. Rheumatology (Oxford) 2007, 46:1460-1465.

35. Roemer FW, Khrad H, Hayashi D, Jara H, Ozonoff A, Fotinos-Hoyer AK, Guermazi A: Volumetric and semiquantitative assessment of MRIdetected subchondral bone marrow lesions in knee osteoarthritis: a comparison of contrast-enhanced and non-enhanced imaging. Osteoarthritis and cartilage/OARS, Osteoarthritis Research Society 2010, 18:1062-1066.

36. Zhao J, Li X, Bolbos Rl, Link TM, Majumdar S: Longitudinal assessment of bone marrow edema-like lesions and cartilage degeneration in osteoarthritis using 3 T MR T1rho quantification. Skeletal Radiol 2010, 39:523-531.

37. Bining HJ, Santos R, Andrews G, Forster BB: Can T2 relaxation values and color maps be used to detect chondral damage utilizing subchondral bone marrow edema as a marker? Skeletal Radiol 2009, 38:459-465.

38. Roemer FW, Bohndorf K: Long-term osseous sequelae after acute trauma of the knee joint evaluated by MRI. Skeletal Radiol 2002, 31:615-623.

39. Buck RJ, Wyman BT, Hellio Le Graverand MP, Hudelmaier M, Wirth W, Eckstein F: Osteoarthritis may not be a one-way-road of cartilage loss comparison of spatial patterns of cartilage change between osteoarthritic and healthy knees. Osteoarthritis Cartilage 2010, 18:335.

\section{Pre-publication history}

The pre-publication history for this paper can be accessed here: http://www.biomedcentral.com/1471-2474/12/217/prepub

doi:10.1186/1471-2474-12-217

Cite this article as: Driban et al: Quantitative bone marrow lesion size in osteoarthritic knees correlates with cartilage damage and predicts longitudinal cartilage loss. BMC Musculoskeletal Disorders 2011 12:217.

\section{Submit your next manuscript to BioMed Central and take full advantage of:}

- Convenient online submission

- Thorough peer review

- No space constraints or color figure charges

- Immediate publication on acceptance

- Inclusion in PubMed, CAS, Scopus and Google Scholar

- Research which is freely available for redistribution

Submit your manuscript at www.biomedcentral.com/submit
C Biomed Central 